\title{
Lipid Monolayers and Adsorbed Polyelectrolytes with Different Degrees of Polymerization
}

\author{
Thomas Ortmann, Heiko Ahrens, Frank Lawrenz, Andreas Gröning, Peter Nestler, Jens-Uwe Günther, \\ and Christiane A. Helm*
}

Institut für Physik, Ernst-Moritz-Arndt Universität, Felix-Hausdorff-Straße 6, D-17487 Greifswald, Germany

\section{Supporting Information}

ABSTRACT: Polystyrene sulfonate (PSS) of different molecular weight $M_{\mathrm{w}}$ is adsorbed to oppositely charged DODAB monolayers from dilute solutions $(0.01 \mathrm{mmol} / \mathrm{L})$. PSS adsorbs flatly in a lamellar manner, as is shown by X-ray reflectivity and grazing incidence diffraction (exception: PSS with $M_{\mathrm{w}}$ below $7 \mathrm{kDa}$ adsorbs flatly disordered to the liquid expanded phase). The surface coverage and the separation of the PSS chains are independent of PSS $M_{\mathrm{w}}$. On monolayer compression, the surface charge density increases by a factor of 2 , and the separation of the PSS chains decreases by the same factor. Isotherms show that on increase of PSS $M_{\mathrm{w}}$ the transition pressure of the LE/LC (liquid expanded/liquid condensed) phase transition decreases. When the contour length exceeds the persistence length $(21 \mathrm{~nm})$, the transition pressure is low and constant. For low- $M_{\mathrm{w}}$ PSS $(<7 \mathrm{kDa})$ the LE/LC transition of the lipids and the disordered/ordered transition of adsorbed PSS occur simultaneously, leading to a maximum in the contour length dependence of the transition enthalpy. These findings show that lipid monolayers at the air/water interface are a suitable

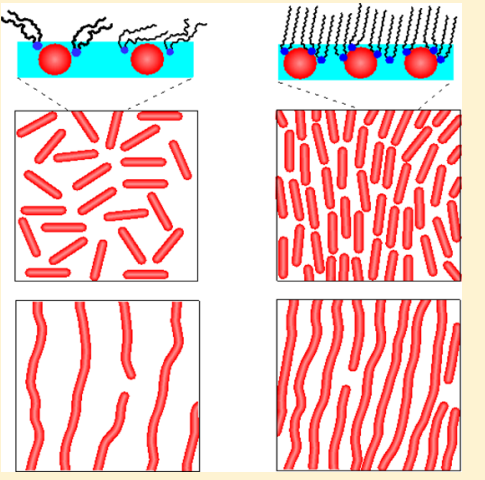
model substrate with adjustable surface charge density to study the equilibrium conformation of adsorbed polyelectrolytes as well as their interactions with a model membrane.

\section{INTRODUCTION}

Traditional Langmuir monolayers at the air/water interface are formed from amphiphilic molecules. They contain lipid heads that anchor onto the water subphase and hydrophobic tails that point toward the air. ${ }^{1}$ These monolayers have gained interest in the field of chemistry, physics, and life science. ${ }^{2-4}$ They serve as model systems for studying two-dimensional (2-D) phase transitions and ordering ${ }^{5,6}$ as well as templates for biological reactions. ${ }^{7,8}$ Also, insight into the equilibrium conformation of adsorbed macromolecules (proteins, DNA, or nanoparticles $)^{9-11}$ and into their interactions with the model membrane ${ }^{12}$ can be gained.

Most water-soluble polymers are charged; they are so-called polyelectrolytes (PEs). They adsorb onto oppositely charged surfaces. The surface coverage increases on increase of the PE concentration in the solution until saturation is reached. ${ }^{13}$ If linear PEs are adsorbed from salt-free solutions, the electrostatic interaction between the charges along the chain has a large amplitude and is long-ranged. The PEs adsorb in a flat conformation. $^{14,15}$ At high PE concentration in solution, the surface coverage is high. The chains are close to each other, and short-ranged interactions between the chains are important, which adsorb flatly disordered. The surface charge of the substrate is reversed due to the high surface coverage of the PEs. ${ }^{13,16-18}$ At low PE concentration, the surface coverage is low. The adsorbed PEs do not compensate for the surface charge of the substrate. The adsorbed chains repel each other electrostatically and adsorb either in a disordered phase or in a lamellar manner. ${ }^{19,20}$ In the two-dimensional lamellar phase the chains are aligned parallel to each other. ${ }^{21,22}$

However, the theory of linear polymers can only be used, when the contour length $L_{\mathrm{K}}$ of the adsorbing PE exceeds the persistence length $L_{\mathrm{P}}$. Informally, molecules with a contour length $L_{\mathrm{K}}$ shorter than the persistence length $L_{\mathrm{P}}$ can be described as rigid polymer chains or elastic rods. Compared to neutral polymers, polyelectrolytes have an increased persistence length $L_{\mathrm{P}}$ due to electrostatic monomer-monomer repulsion. $^{22-25}$ AFM measurements of polystyrene sulfonate (PSS) in the two-dimensional lamellar phase adsorbed to very weakly charged surfaces ${ }^{19}$ show that PSS has a persistence length $L_{\mathrm{P}}$ of $340 \AA$ (which would be equal to the contour length for a molecular weight of $27 \mathrm{kDa}$ ). Note that the steric persistence length of PSS is much lower $\left(L_{\mathrm{P}, \text { steric }}=30 \AA\right)$. To find out how the size of the adsorbed macromolecule affects the lipid monolayer and the 2-D lamellar phase, the molecular weight, i.e., the contour length $L_{\mathrm{K}}$ of the polyelectrolyte is varied. ${ }^{26}$

For large contour lengths $L_{\mathrm{K}}$ exceeding the persistence length $L_{\mathrm{P}}$, the entropic in-plane interactions of the adsorbed polymers need to be taken into account. ${ }^{27}$ The possible conformations of the adsorbed polymers are confined to the surface plane, and additionally a repulsive free energy contribution coming from steric interactions between stiff polymers has to be considered. $^{21,25,28}$ The 2-D lamellar phase is characterized by the

Received: June 8, 2013

Revised: April 16, 2014

Published: June 3, 2014 
separation between the aligned polyelectrolyte chains $d_{\mathrm{PSS}}$, which is a measure of the $\mathrm{PE}$ coverage. $L_{\mathrm{D}}$ denotes the deflection length and is defined as $L_{\mathrm{D}}{ }^{3} \cong{d_{\mathrm{PSS}}}^{2} L_{\mathrm{P}}$. In the case of $L_{\mathrm{K}}>L_{\mathrm{D}}>d_{\mathrm{PSS}}{ }^{28}$ one has to apply the statistics of a wormlike chain in a cylindrical pore of diameter $d_{\mathrm{PSS}}$, which is always shorter as the persistence length $L_{\mathrm{p}}$. Then, the repulsive entropic energy per unit area $\Delta F / k_{\mathrm{B}} T \simeq 1 /\left(d_{\mathrm{PSS}}{ }^{5 / 3} L_{\mathrm{D}}{ }^{1 / 3}\right)$ $\ln \left(L_{\mathrm{D}} / d_{\mathrm{PSS}}\right)$ is independent of the degree of polymerization $N$ and depends only on the surface coverage of the polyelectrolyte (measured by $d_{\mathrm{PSS}}$ ). Concluding, if the contour length $L_{\mathrm{K}}$ exceeds the persistence length $L_{\mathrm{P}}$, then the exact value of the contour length $L_{\mathrm{K}}$ is irrelevant.

When the contour length $L_{\mathrm{K}}$ is shorter than the persistence length $L_{\mathrm{P}}$, the rigid PEs can be described as elastic rods with length $L_{\mathrm{K}}$. In the 2-D lamellar phase, these rods are aligned parallel to each other. However, it is not known at which surface coverage alignment occurs. Theories predict for elastic rods within a surface plane an "isotropic/nematic" phase transition. ${ }^{29,30}$ The nematic phase is a two-dimensional lamellar phase.

Lipid monolayers at the air/water interface serve as model substrate with adjustable surface charge density. Surface pressure area $(\pi-A)$ isotherms are used to elucidate the phase behavior of the lipid monolayers. Different phases can be distinguished: gaseous, fluid (liquid expanded, LE), and condensed or solid phases (liquid condensed, LC) can be formed, dependent on the molecular area and the temperature. The LE/LC phase transition appears as a plateau in the isotherm; its onset occurs at a surface pressure $\pi_{\mathrm{c}}{ }^{31} \pi_{\mathrm{c}}$ is a measure of the headgroup repulsion; an increase of the repulsion increases $\pi_{c}$ and a decrease of the repulsion or an attraction decreases $\pi_{c^{c}}{ }^{2,9,32}$ Changes of the phase transition enthalpy and its temperature dependence are attributed to different interactions of the alkyl tails of the lipid; for instance, the formation of a hydrogen-bonding network in the fluid phase decreases the phase transition enthalpy and its temperature dependence dramatically. ${ }^{33}$

It is far from obvious how the phases and the LE/LC phase transition of a lipid monolayer are affected by the adsorbed macromolecules. To shed some light on the question, we use a system where electrostatic interactions between the macromolecules and the lipid monolayer dominate. Lipids with small head groups are chosen to further simplify the system, i.e., to avoid changes of the headgroup conformation. As a lipid, cationic dioctadecyldimethylammonium (DODA) is chosen since it has a very small headgroup and its LC phase is not influenced by PSS adsorption (oblique lattice and tilted alkyl chains). ${ }^{20}$ As typical charged macromolecules we use PSS, because we know that the 2-dimensional lamellar/flatly disordered transition occurs at concentrations of $0.1-1$ $\mathrm{mmol} / \mathrm{L}$ (with respect to the monomer concentration). ${ }^{20}$ We use a concentration of $0.01 \mathrm{mmol} / \mathrm{L}$ because we know that PSS with large molecular weight adsorbs in the 2-D lamellar conformation.

The molecular weight of PSS and thus its contour length $L_{\mathrm{K}}$ are varied by 3 orders of magnitude. To study the phase transition pressure $\pi_{\mathrm{c}}$ and the transition enthalpy $\Delta H$, the temperature dependence of the isotherms is analyzed. In the two-dimensional lamellar phase, the separation of the aligned polyelectrolyte chains $d_{\mathrm{PSS}}$ and the order of the hydrophobic alkyl tails are measured with grazing incidence X-ray diffraction. The surface coverage of PSS in the disordered 2-D phase is determined with X-ray reflectivity.

\section{EXPERIMENTAL SECTION}

Materials. DODA bromide is from Avanti, Alabaster, AL. PSS sodium salt with different molecular weight $M_{\mathrm{w}}$ is used (3.7, 4.4, 6.5, 8.6, 16.8, 77.4, and $168 \mathrm{kDa}$ with PDI $\leq 1.1$ and degree of sulfonation 97\% from Polymer Standards Service, Mainz, Germany; $M_{\mathrm{w}}=1330$ $\mathrm{kDa}$ with PDI $=1.2$ is from BASF, Ludwigshafen, Germany). From $M_{\mathrm{w}}$, one obtains $N=4.82 \cdot M_{\mathrm{w}}$ (with $M_{\mathrm{w}}$ in $\mathrm{kDa}$ ) and $L_{\mathrm{K}}=N \cdot 2.56 \AA$. A table of $N, L_{\mathrm{K}}$, and $M_{\mathrm{w}}$ is in the Supporting Information.

Langmuir Trough and Isotherms. Surface pressure isotherms $(\pi-A$ isotherms) are measured on a Teflon trough (Riegler \& Kirstein, Potsdam, Germany). The surface pressure is recorded with a Wilhelmy-type pressure measuring system using filter paper as plate with an accuracy better $0.1 \mathrm{mN} / \mathrm{m}$. The area of the trough is $3 \times 30$ $\mathrm{cm}^{2}$. DODA is dissolved in chloroform/methanol (3:1) solution (0.14 $\mathrm{mmol} / \mathrm{L})$. The solution is spread with a $100 \mu \mathrm{L}$ syringe. The monolayers are compressed at a rate of $3 \AA^{2}$ per molecule and minute after allowing $10 \mathrm{~min}$ for the evaporation of the solvent. Ultrapure water from a Milli- $Q$ system with a specific resistance above $18 \mathrm{M} \Omega$. $\mathrm{cm}$ is used. Always, the subphase contains PSS. Always, the PSS concentration is set to $10 \mu \mathrm{mol} / \mathrm{L}$ (with respect to the monomer concentration) and kept constant. The temperature is controlled by a thermostat (DC-30 Thermo-Haake, Haake-Technik, Karlsruhe, Germany). All isotherms shown are obtained during the first compression of the monolayer.

Grazing Incidence X-ray Diffraction. Grazing-incidence diffraction measurements were performed at the liquid surface diffractometer at the undulator beamline BW1 in HASYLAB (DESY, Hamburg, Germany). ${ }^{34}$ With this technique, a monochromatic X-ray beam $(\lambda=1.303 \AA)$ strikes the surface at grazing-incidence angle $\alpha_{\mathrm{i}}=$ $0.85 \alpha_{c}\left(\alpha_{c}=0.13^{\circ}\right.$ is the critical angle for total reflection). The intensity of the Bragg peaks is measured by a linear position sensitive detector (PSD; OED-100-M, Braun, Garching, Germany) as a function of the vertical scattering angle $\alpha_{\mathrm{f}}{ }^{20,35}$ The resolution of $0.09^{\circ}$ of the in-plane scattering angle $2 \theta$ is given be the Soller collimator in front of the PSD. The measurements presented in this paper were recorded at five different beam times; each time a new alignment was used. Therefore, the absolute intensities cannot be compared quantitatively.

According to the geometry of diffraction, the scattering vector $\mathbf{Q}$ has an in-plane component, $Q_{x y}=2 \pi / \lambda\left(\cos ^{2} \alpha_{\mathrm{i}}+\cos ^{2} \alpha_{\mathrm{f}}-2 \cos \alpha_{\mathrm{i}} \cos \alpha_{\mathrm{f}}\right.$ $\cos 2 \theta)^{1 / 2}$ and an out-of-plane component $Q_{z}=2 \pi / \lambda\left(\sin \alpha_{\mathrm{i}}+\sin \alpha_{\mathrm{f}}\right)$. The positional correlation length $\xi$ can be estimated from the full width at half-maximum (fwhm). For an exponential decay of positional correlation as observed in liquid crystals, corresponding to a Lorentzian as a Bragg profile, one obtains $\xi=2 /$ fwhm $\left(Q_{x y}\right)$.

The position-resolved scans were corrected for illuminated area. Model peaks are assumed to be Lorentzians in the in-plane direction and Gaussians in the out-of-plane direction and are fitted to corrected intensities. The lattice spacings are obtained from the in-plane diffraction data.

X-ray Reflectivity. X-ray reflectivity measurements were carried out with a homemade setup with a $\mathrm{Cu}$ anode (wavelength $\lambda=1.54 \AA$ ), which is described in detail elsewhere. ${ }^{36}$ The setup is equipped with a temperature-controlled Langmuir trough, which is enclosed in a sealed container. The specular X-ray reflectivity (XR) data collection is performed using a $\mathrm{NaI}$ scintillator detector. The $\mathrm{X}$-ray reflectivity was measured with the geometry $\alpha_{\mathrm{i}}=\alpha_{\mathrm{f}} \equiv \alpha$, where $\alpha_{\mathrm{i}}$ is the vertical incidence angle and $\alpha_{\mathrm{f}}$ is the vertical exit angle of the reflected X-rays. XR data are collected as a function of the incidence angle $\alpha_{\mathrm{i}}$, varied in the range of $0.01^{\circ}-5^{\circ}$, corresponding to a range of $0.01-0.6 \AA^{-1}$ of the vertical scattering vector component $Q_{z}$.

The technique provides information on the electron density variation perpendicular to the surface with subnm resolution, since the index of refraction depends linearly on the electron density $\rho$ (Thompson radius $r_{0}=2.8 \times 10^{-5} \AA$ ) , $n=1-r_{0} \rho \lambda^{2} / 2 \pi{ }^{37}$ Since the refractive index is slightly less than 1 (ca. $10^{-5}$ ), dynamic effects (e.g., multiple refraction) contribute only at very small angles of incidence $\alpha_{\mathrm{i}}$ to the reflected intensity $R$. Above about twice the critical angle the 
reflectivity $R$ (normalized to the Fresnel reflectivity $R_{\mathrm{F}}$ of the ideally smooth surface) can be described by the kinematic approximation ${ }^{38}$

$$
\frac{R}{R_{\mathrm{F}}}=\left|\frac{1}{\rho_{\text {sub }}} \int_{0}^{\infty} \frac{\mathrm{d} \rho}{\mathrm{d} z} \exp \left(i Q_{z} z\right) \mathrm{d} z\right|^{2}
$$

where $z$ is vertical distance from solution surface, $\rho_{\text {sub }}$ is the electron density of the subphase $\left(0.334 e \AA^{-3}\right.$ with $e$ the elementary charge $)$, $\mathrm{d} \rho / \mathrm{d} z$ is the gradient of the electron density along the surface normal, and $Q_{z}=4 \pi / \lambda \sin \left(\alpha_{\mathrm{i}}\right)$ is the wave vector transfer normal to the surface.

The background scattering from the subphase is measured at $\alpha_{\mathrm{f}}-\alpha_{\mathrm{i}}$ $=1^{\circ}$ and subtracted from the signal measured at $\alpha_{\mathrm{f}}=\alpha_{\mathrm{i}}$. The electron density profile has been obtained by two complementary strategies: (i) The electron density profile is determined with a model-independent method. ${ }^{39,40}$ From the experimentally observed reflectivity curve, the corresponding profile correlation function is estimated via indirect Fourier transformation. For this profile correlation function the matching electron density profile is then derived by square-root deconvolution. No a priori assumptions on the shape of the electron density profile have to be made. (ii) The slab model is used, and individual slabs can be identified with certain molecular properties of the layer. For the least-squares fits as few independent parameters as possible are used. ${ }^{35}$ The criterion for a satisfying interpretation of the XR data is the matching of the scattering profiles resulting from the two different modeling approaches.

\section{RESULTS}

Isotherms and Thermodynamics. Figure 1 shows a series of $\pi-A$ isotherms of DODA with adsorbed PSS with different

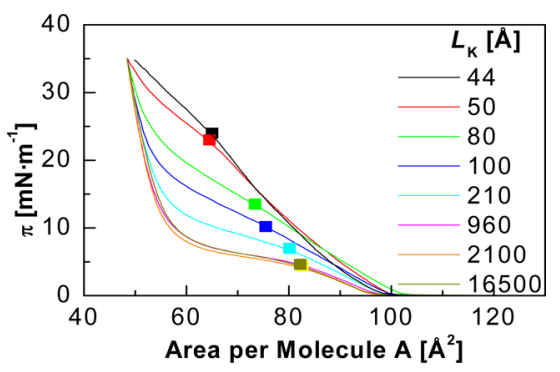

Figure 1. Surface pressure-area isotherms of DODA on $0.01 \mathrm{mmol} / \mathrm{L}$ PSS solution (with respect to the monomer concentration at $18{ }^{\circ} \mathrm{C}$. Varied is the contour length of PSS (indicated). The squares symbolize the transition pressure $\pi_{\mathrm{c}}$.

contour length, while the temperature is kept constant $\left(18^{\circ} \mathrm{C}\right)$. Each isotherm has the typical shape known from fatty acid or phospholipid monolayers: ${ }^{1}$ The fluid phase of the lipids is marked by an increase in surface pressure $\pi$ on monolayer compression. A break in the slope at $\pi_{\mathrm{c}}$ marks the onset of the LE/LC phase transition, which shows the typical plateau indicative of the coexistence of lipids in the LE and LC phase. ( $\pi_{\mathrm{c}}$ is determined by fitting two straight lines to the isotherm: one at the end of the LE phase and one at the beginning of the plateau region. $\pi_{\mathrm{c}}$ is assigned to the intersection; cf. Figure $S 1$ in the Supporting Information.) At the end of the coexistence region, the lipids are in the LC phase and $\pi$ increases steeply. With increasing contour length $L_{\mathrm{K}}$ of the PSS chain, the transition pressure $\pi_{\mathrm{c}}$ decreases, until it levels off at $L_{\mathrm{K}} \approx 300 \AA$. Two features are remarkable: (i) $\pi_{\mathrm{c}}$ levels off when the contour length $L_{\mathrm{K}}$ approaches $210-300 \AA$, and (ii) the variation of $\pi_{\mathrm{c}}$ is unusually large, $\Delta \pi_{\mathrm{c}}=20 \mathrm{mN} / \mathrm{m}$, demonstrating a condensing effect of adsorbed polyelectrolytes.

The temperature dependence of the isotherms is measured for each PSS with its specific contour length $L_{\mathrm{K}}$. Representative examples are shown in Figure S2 of the Supporting Information. As expected, $\pi_{\mathrm{c}}$ increases on heating the subphase. However, one feature is unusual: For the long contour length $\left(L_{\mathrm{K}}=16500 \AA\right)$ a much weaker temperature dependence of $\pi_{\mathrm{c}}$ is observed than for the short one $\left(L_{\mathrm{K}}=80 \AA\right)$.

The temperature dependence of $\pi_{c}$ is quantified in Figure 2 . For all contour lengths $\pi_{c}$ increases linearly with the temperature; however, the slope $\partial \pi_{\mathrm{c}} / \partial T$ varies. It is largest for short contour lengths $\left(\partial \pi_{\mathrm{c}} / \partial T=1.5 \mathrm{mN} \mathrm{m}^{-1} \mathrm{~K}^{-1}\right.$ for $L_{\mathrm{K}}=$ $44,50 \AA)$. On increase of the contour length $L_{\mathrm{K}}$ up to $210 \AA$, the slope $\partial \pi_{\mathrm{c}} / \partial T$ decreases linearly by a factor of 2 and then it is constant.

The decrease of $\pi_{\mathrm{c}}$ is pronounced $\left(20 \mathrm{mN} / \mathrm{m}\right.$ at $\left.18{ }^{\circ} \mathrm{C}\right)$. Clearly, adsorption of PSS has a condensing effect, which increases with contour length $L_{\mathrm{K}}$ (up to a threshold value of $\geq 210 \AA$ ). Presumably, the electrostatic repulsion between the lipid head groups is reduced or even turned into an attraction. The binding enthalpy $H_{\mathrm{PSS}}(A)$ depends on the DODA molecular area (inversely proportional to the monolayer surface charge density) and the polyelectrolyte coverage. The shift of the phase transition pressure is ${ }^{32}$

$$
\Delta \pi_{\mathrm{c}, \mathrm{PSS}}=\frac{H_{\mathrm{PSS}}\left(A_{\mathrm{s}}\right)-H_{\mathrm{PSS}}\left(A_{\mathrm{fl}}\right)}{A_{\mathrm{fl}}-A_{\mathrm{s}}}
$$

We find that $\pi_{\mathrm{c}}$ decreases, $\Delta \pi_{\mathrm{c}, \mathrm{PSS}}<0$, and $H_{\mathrm{PSS}}\left(A_{\mathrm{s}}\right)-H_{\mathrm{PSS}}\left(A_{\mathrm{fl}}\right)$ $<0$. The effect is large, $\Delta \pi_{\mathrm{c}}=20 \mathrm{mN} / \mathrm{m}$ is observed $(T=18$ $\left.{ }^{\circ} \mathrm{C}\right)$. Binding of divalent ions causes a large shift of the surface pressure; the change is attributed to the reduction of the electrostatic repulsion between the head groups or even an ionmediated attraction. ${ }^{41,42}$ Reversely, an increase of the surface charge density due to removal of bound counterions fluidizes the monolayer, values up to $\Delta \pi_{\mathrm{c}}=13 \mathrm{mN} / \mathrm{m}$ were reported,. ${ }^{32}$ These shifts of the transition pressure described in the literature are smaller than the $20 \mathrm{mN} / \mathrm{m}$ observed with DODA/PSS.

Additionally, the compression of the adsorbed polymer might affect the surface pressure. Transitions between different phases of polymeric monolayers have been observed in the past. $^{27,43,44}$ If the polymer monolayer undergoes a first-order transition, a plateau region in the isotherm is observed and a transition enthalpy can be determined. In some cases, the surface pressure of the disordered-to-ordered phase transition decreases with increasing molar mass for a given temperature. This trend is consistent with free volume effects; i.e., polymers with smaller molecular mass have a higher degree of molecular motion. ${ }^{45}$ Quantitatively, the reported decrease of the transition pressure $\pi_{\mathrm{c}}$ is small, between 0 and $2 \mathrm{mN} / \mathrm{m} .{ }^{27}$ This is an order of magnitude smaller than the changes of DODA monolayers, which can be induced by increasing the contour length of PSS. Therefore, the pronounced shift of the transition pressure $\pi_{c}$ is attributed to an in-plane attraction between the lipid molecules which is mediated by the adsorbed PSS. Thus, the adsorbed polyelectrolyte can be described as a cloud of small counterions which are connected by the polymer backbone.

To better understand the LE/LC phase transition of the lipid monolayer, the transition enthalpy $\Delta H$ is calculated according to the two-dimensional Clausius-Clapeyron equation

$$
\Delta H=\frac{\partial \pi_{\mathrm{c}}}{\partial T} T\left(A_{\mathrm{fl}}-A_{\mathrm{s}}\right) N_{\mathrm{A}}
$$

where $T$ denotes the temperature, $N_{\mathrm{A}}$ is the Avogradro constant, $A_{\mathrm{fl}}$ is the molecular area in the LE phase at the onset of the phase transition, and $A_{s}$ is the molecular area in the 

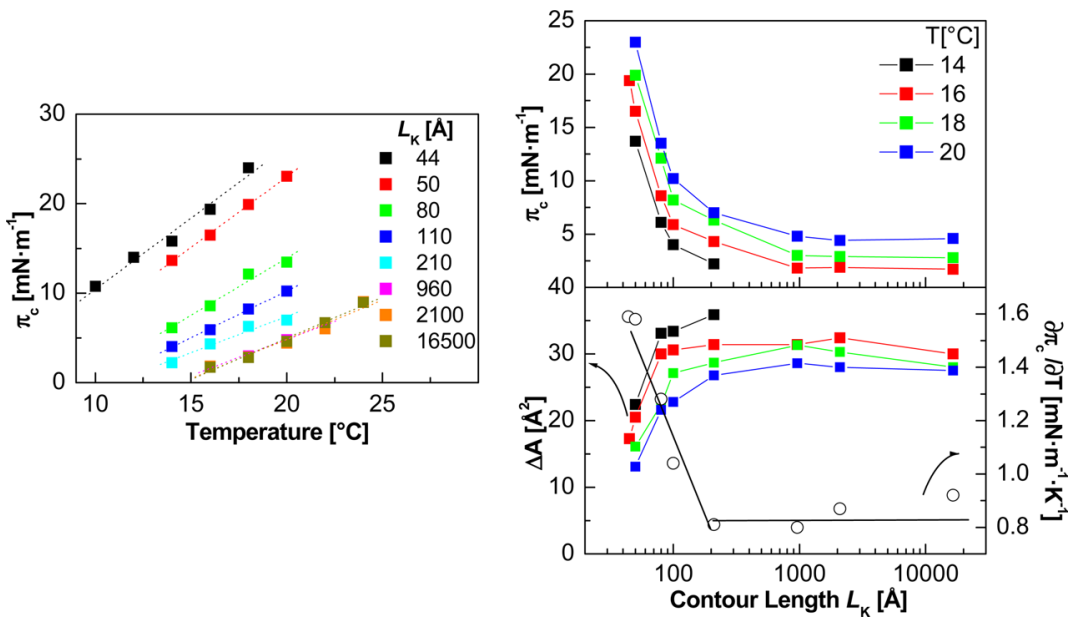

Figure 2. Left: temperature dependence of the transition pressure $\pi_{\mathrm{c}}$ of DODA on $0.01 \mathrm{mmol} / \mathrm{L}$ (in monomer units) PSS solution; varied is the contour length $L_{\mathrm{K}}$ (indicated). The straight lines are linear fits; the slope gives $\partial \pi_{\mathrm{c}} / \partial T$. Right: contour length dependence of the transition pressure $\pi_{\mathrm{c}}$ (top) and of the change of the DODA molecular area at the LE/LC phase transition, $\Delta A=A_{\mathrm{fl}}-A_{\mathrm{s}}$, together with $\partial \pi_{\mathrm{c}} / \partial T$ (bottom) at the temperatures indicated.

LC phase. The parameters that influence the transition enthalpy $\Delta H$ are shown in Figure 2: The transition pressure $\pi_{\mathrm{c}}$ decreases on increase of the contour length $L_{\mathrm{K}}$ up to $L_{\mathrm{K}} \approx$ 210-300 $\AA$, and then it levels off. Similarly, the change of the DODA molecular area during the LE/LC phase transition $(\Delta A$ $=A_{\mathrm{fl}}-A_{\mathrm{s}}$ ) increases on increase of the contour length up to $L_{\mathrm{K}}$ $=210 \AA$, and then it is constant. On heating of the solution $\pi_{\mathrm{c}}$ increases and $\Delta A$ decreases; however, the respective temperature dependence is essentially unchanged.

For every contour length $L_{\mathrm{K}}, \Delta H$ is lowered by heating (cf. Figure 3 and Figure S3). Qualitatively, this observation agrees

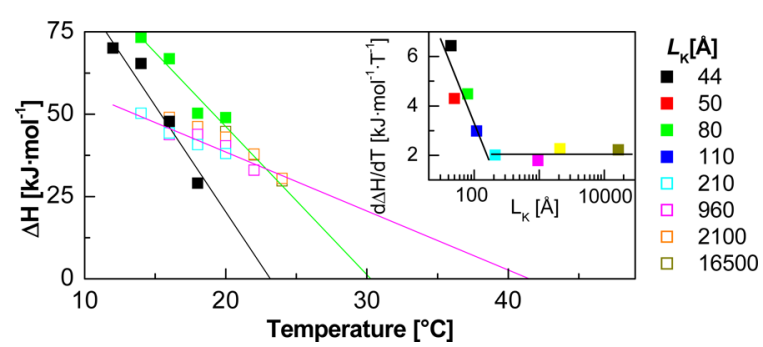

Figure 3. Temperature dependence of the transition enthalpy $\Delta H$ of DODA on $0.01 \mathrm{mmol} / \mathrm{L}$ (with respect to the monomer concentration) PSS solution. Varied is the contour length $L_{\mathrm{K}}$ (indicated). All experimental points are shown in Figure S3. The straight lines are linear fits. For each contour length, the same color is used for the data points and the fitted line. An exception are the measurements for $L_{\mathrm{K}}=$ 210, 960, 2100, and $16500 \AA$; these points almost superimpose, and the fitted line is pink. Inset: temperature dependence of the transition enthalpy $\partial \Delta H / \partial T$ as function of the contour length $L_{\mathrm{K}}$.

with earlier observations of simple lipid monolayers. ${ }^{2,31}$ However, the contour length dependence of $\partial \Delta H / \partial T$ shows a constant decrease down to a contour length of $\approx 210 \AA$, and then it levels off. (The dependence of $\Delta H$ on the transition pressure $\pi_{\mathrm{c}}$ is shown in Figure S3.) Actually, $\Delta H$ depends on the temperature and the contour length in a nonmonotonous way which is best seen as a three-dimensional plot viewed from different angles (cf. Figure 4, left).

From the temperature dependence of $\Delta H$ the upper temperature $T_{\text {up }}$, above which the monolayer cannot be compressed into a condensed state, is determined by extrapolating $\Delta H$ to zero (cf. Figure 3 and Figure S3). Similarly, $T_{\text {down }}$, the temperature below which no fluid phase exists, is determined by extrapolating $\pi_{\mathrm{c}}$ to zero (cf. Figure 2, left). The temperature interval in which the isotherms show a LE/LC phase transition is limited by $T_{\text {down }}$ and $T_{\text {up }}$. To balance the condensing effect of the polyelectrolyte adsorption, this interval shifts to larger temperatures on increase of the contour length $L_{\mathrm{K}}$ (cf. Figure 4). Both $T_{\text {down }}$ and $T_{\text {up }}$ increase with $L_{\mathrm{K}}$ up to $L_{\mathrm{K}}=210 \AA$, and then they are constant. Interestingly, the temperature interval in which the isotherms show a $\mathrm{LE} / \mathrm{LC}$ phase transition is rather independent of $L_{\mathrm{K}}\left(\right.$ about $\left.25^{\circ} \mathrm{C}\right)$; it just shifts.

Note that $T_{\text {up }}$ and $T_{\text {down }}$ decrease because of the shift in the transition pressure $\pi_{c}$. This shift is attributed mainly to polyelectrolyte/lipid interactions in the direction perpendicular to the monolayer. However, the transition enthalpy measures changes of the lipid/lipid and the polymer/polymer interactions within the lipid or polymer plane, respectively. In a first approximation, the in-plane and out-of-plane interactions are independent of each other.

At $18{ }^{\circ} \mathrm{C}$, the monolayer shows a $\mathrm{LE} / \mathrm{LC}$ phase transition for all contour lengths. Quantitatively, $\Delta H$ varies by about a factor of 3 (cf. Figure 4). At this temperature, the contour length dependence is not systematic: $\Delta H$ is largest at for $L_{\mathrm{K}}=80 \AA$; on further increase of $L_{\mathrm{K}}$, the transition enthalpy $\Delta H$ decreases. When the contour length exceeds $210 \AA$ ( $\left.L_{\mathrm{K}} \geq 210 \AA\right)$, all values for $\Delta H$ superimpose within resolution; the contour length is no longer an important parameter.

In order to understand the contour length dependence of the transition enthalpy, we first want to determine the persistence length of adsorbed PSS from all thermodynamic parameters described so far. These are the phase transition pressure $\pi_{\mathcal{c}}$ its temperature dependence $\partial \pi_{c} / \partial T$, the phase transition enthalpy $\Delta H$, and the temperatures above which only the LE phase or below which only the LC phase exist, $T_{\text {up }}$ and $T_{\text {down }}$. All these parameters shift with the contour length monotonously up to $L_{\mathrm{K}} \approx 210 \AA$, and then they are constant. Actually, the values of $\partial \pi_{\mathrm{c}} / \partial T, \Delta H$, and $T_{\text {down }}$ are all identical for the contour lengths $210,960,2100$, and $16500 \AA$. Therefore, we conclude $L_{\mathrm{P}} \approx 210$ $\AA$. According to Odjik, ${ }^{28}$ the repulsive entropic energy (per unit area) of a polymer is independent of the degree of 

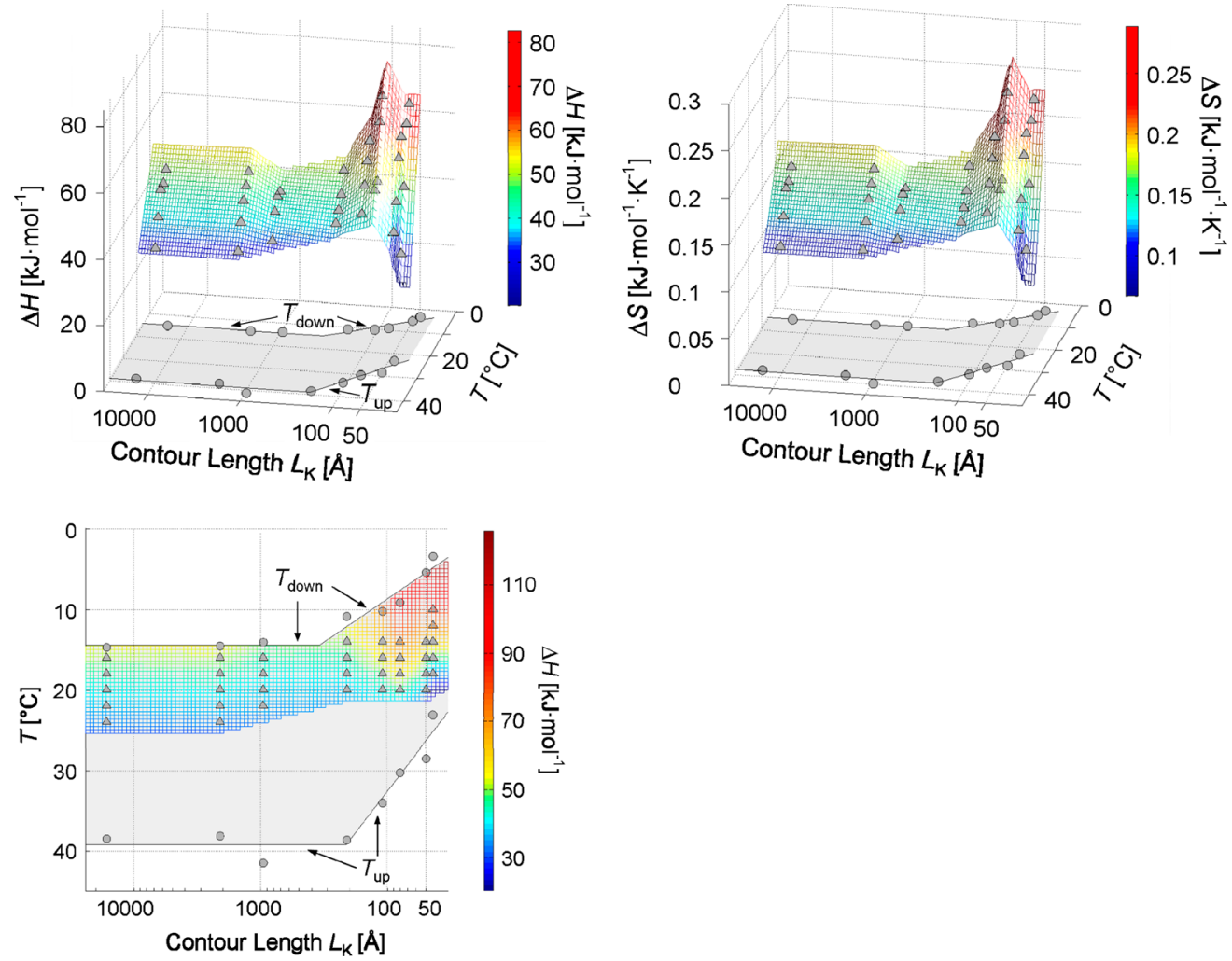

Figure 4. Three-dimensional contour plot of the transition enthalpy $\Delta H$ (left, bottom and top) and the transition entropy $\Delta S$ (right) as a function of the temperature $T$ and the contour length $L_{\mathrm{K}}$. The triangles symbolize measurements of the enthalpy (cf. Figure 3 and Figure S3). The plotted grid is calculated according to a triangle-based linear interpolation of the measured data. The color of the grid symbolizes the value of $\Delta H$ or $\Delta S$ (see scale on the right). The circles indicate the contour length dependence of the critical temperature, $T_{\text {up }}$, and the lower temperature, $T_{\text {down }}$, of DODA monolayers on $0.01 \mathrm{mmol} / \mathrm{L}$ PSS solutions (in monomer units). Only if $T_{\text {down }} \leq T \leq T_{\text {up }}$, the lipid monolayer with the adsorbed PSS shows a LE/ LC phase transition (gray area).

polymerization $N$ and depends only on the surface coverage. Therefore, we conclude that the persistence length is $L_{\mathrm{P}} \approx 210$ $\AA$. If the contour length exceeds the persistence length of 210 $\AA$, then PSS can be described as a flexible chain. Otherwise, PSS is described as a elastic rod.

Compared to neutral polymers, polyelectrolytes have an increased persistence length $L_{\mathrm{P}}$ due to electrostatic monomermonomer repulsion. ${ }^{22-24}$ In solution, one obtains

$$
L_{\mathrm{P}}=L_{\mathrm{P}, \text { steric }}+\left(\tau^{2} l_{\mathrm{B}}\right) /\left(4 \kappa^{2}\right)
$$

$L_{\mathrm{P}, \text { steric }}$ is the steric persistence length (PSS: $L_{\mathrm{P}, \text { steric }}=30 \AA$ ), and $\tau$ is the charge density per unit length (PSS: $\tau=1 / 2.5 \AA$ ). $l_{\mathrm{B}}$ is the Bjerrum length ( $7 \AA$ for water at room temperature), and $\kappa^{-1}$ is the Debye length $\left(\kappa=\left(8 \pi c_{\text {salt }} l_{\mathrm{B}}\right)^{1 / 2}\right.$ with $c_{\text {salt }}$ the concentration of monovalent ions). At the interface, $L_{\mathrm{P}}=210 \AA$ corresponds to an effective salt concentration $c_{\text {salt,eff }}=0.014$ $\mathrm{mol} / \mathrm{L}$. The salt concentration in solution is $2-3$ orders of magnitude smaller than the effective one deduced from the persistence length of adsorbed PSS. The counterion concentration at a charged surface is increased by $1-2$ orders of magnitude; it is further modified by the in-plane repulsion of adsorbed charged particles. ${ }^{4-48}$ These effects may contribute to the high value of $c_{\text {salt,eff }}$

When the contour length dependence of $\Delta H$ is considered, it is helpful to consider the entropy change $\Delta S$ during the LE/LC phase transition. ${ }^{1}$

$$
\Delta S=\partial \pi_{\mathrm{c}} / \partial T\left(A_{\mathrm{fl}}-A_{\mathrm{s}}\right) N_{\mathrm{A}}=\Delta H / T
$$

Two features are striking: (1) a maximum at $L_{\mathrm{K}}=80 \AA$ and (2) the reduction of $\Delta S$ on further increase of $L_{\mathrm{K}}$ (cf. Figure 4 and Figure S5); $\Delta S$ is small and constant when $L_{\mathrm{K}}$ exceeds $L_{\mathrm{P}}$. The transition entropy $\Delta S$ shows the same contour length dependence as the transition enthalpy $\Delta H$ : a maximum at $L_{\mathrm{K}}$ $=80 \AA$ and constant low values for $L_{\mathrm{K}} \geq L_{\mathrm{P}}=210 \AA$ (cf. Figure 4). First, we will discuss the binding of PSS with large contour lengths, when PSS is a flexible chain. Then, not only $\Delta S$ and $\Delta H$ are low and constant, but also the transition pressure $\pi_{\text {c }}$ To explain the decrease of $\Delta S$ on adsorption of PSS with large contour length $\left(L_{\mathrm{K}}>L_{\mathrm{P}}\right)$, either the LC phase must gain entropy or the LE phase must lose entropy. The structure of the lipids in the LC phase does not change, when PSS is adsorbed, independent of its contour length. Thus, the lipids in the LE phase have to lose entropy when PSS with large contour length is adsorbed. In a simplified picture, a PSS molecule with $n$ charges neutralizes $n$ lipid molecules. With increasing $n$, we find a decreased entropy change $\Delta S$ at the LE/LC phase transition, which may be explained by the partial immobilization of $n$ fluid lipids attracted electrostatically to a polymer with $n$ charged monomers. The effect is small, but it is unambiguous.

In the second step, the maximum of the transition enthalpy $\Delta H$ at $L_{\mathrm{K}}=80 \AA$ is addressed (cf. Figure 4). We considered many thermodynamic parameters. This maximum occurs only at $\Delta H$ and $\Delta S$ which depend on the in-plane interactions of the lipids and the PSS. The out-of-plane interactions between lipid head groups and PSS influence the transition pressure $\pi_{\mathcal{c}}$ which decreases monotonously up to $L_{\mathrm{P}}$ with increasing contour 

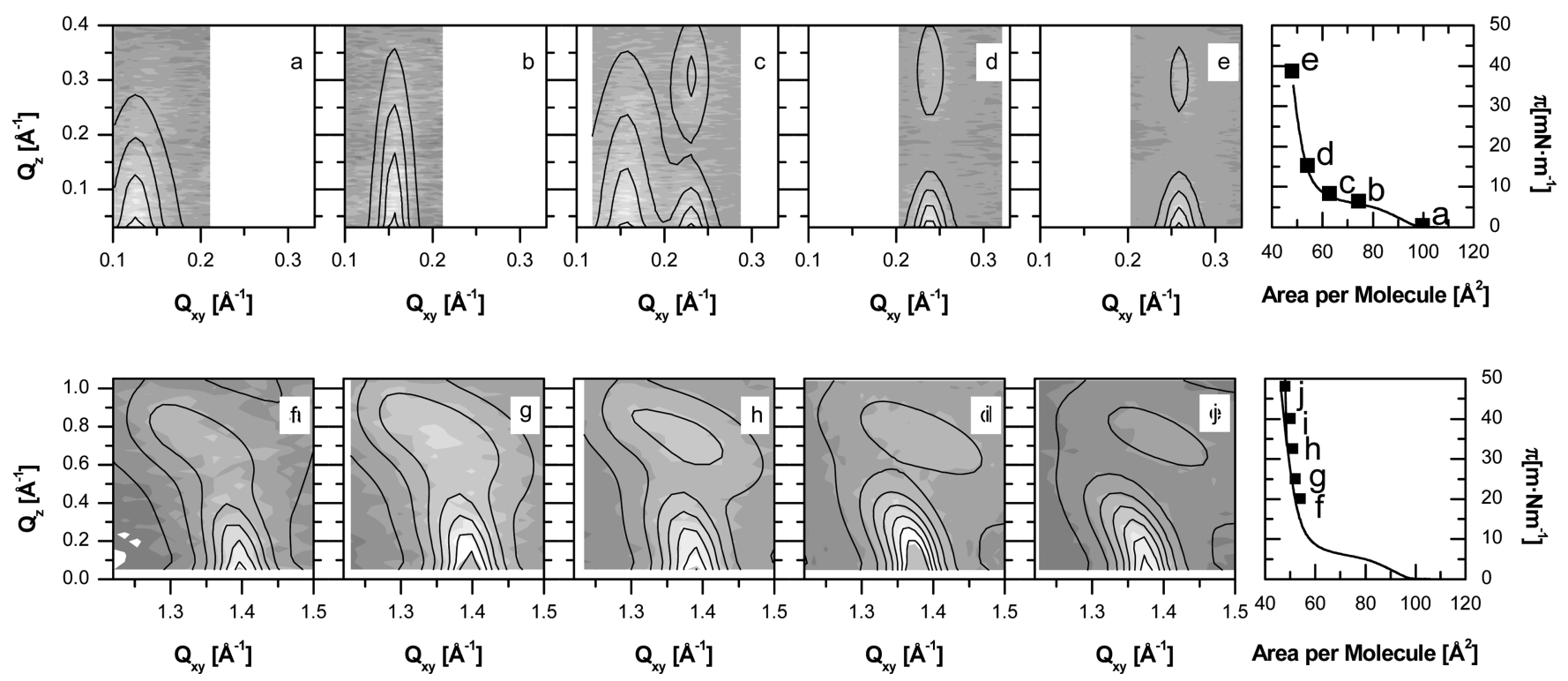

Figure 5. Grazing incidence X-ray diffraction (GID) measurements at the DODA molecular areas indicated in the isotherm on $0.01 \mathrm{mmol} / \mathrm{L}$ PSS (with respect to the monomer concentration) with $L_{\mathrm{K}}=960 \AA . T=20^{\circ} \mathrm{C}$. The top row shows low-angle diffraction peaks attributed to aligned PSS chains: (a, b) While the lipids are in the LE phase, a peak due to the aligned PSS chains is observed. (c) In the LE/LC coexistence region of the lipids, two different PSS peaks are observed. One is attributed to the PSS chains adsorbed to lipids in the LE phase and the other to PSS chains adsorbed to lipids in the LC phase. It is found at larger $Q_{x y}$ and has a structured rod. (d, e) At high surface pressure, the structured peaks remain and shift slightly on monolayer compression. The bottom row shows wide-angle peaks due to the ordered alkyl tails solid lines are during monolayer compression as indicated in the isotherm on the right. Only when the lipids are in the LC phase, wide-angle peaks are observed. Always, background is subtracted and contour plots are calculated from least-squares fits to appropriate models.

length. To understand the subtle changes of $\Delta H$, the monolayer structure is investigated with $\mathrm{X}$-ray techniques.

In-Plane Structure: Grazing Incidence X-ray Diffraction. Small-Angle Peaks. The molecular organization is studied with grazing incidence diffraction measurements along the compression isotherm. A typical measurement for $L_{\mathrm{K}}=960$ $\AA$ is shown in Figure 5. When the lipids are in the fluid phase, always a low-angle peak is observed. It is attributed to aligned PSS chains separated by a distance $d_{\text {PSS }}$. On monolayer compression, the low-angle peak shifts to larger $Q_{x y}$ values (from 0.12 to $0.16 \AA^{-1}$ ), indicating a decrease of $d_{\mathrm{PSS}}$. In the coexistence region, the low-angle peak observed in the fluid phase exhibits a constant position (cf. Figure 5). However, if the monolayer is compressed further and the area fraction of the lipids in the fluid phase decreases, then the low-angle peak loses intensity and eventually disappears. Simultaneously, at larger $Q_{x y}$ values a low-angle peak with an unusual structured rod in $Q_{z}$ direction appears (two peaks at the same $Q_{x y}$ and different $Q_{z}$ positions). Its intensity increases when the monolayer is further compressed. The positions of the two different low-angle peaks never coincide. To be quantitative, no peaks are observed with $Q_{x y}$ positions between 0.181 and 0.230 $\AA^{-1}$. Actually, this statement is true for all contour lengths investigated (see Figure S6). Peak positions smaller than 0.181 $\AA^{-1}$ are observed when the lipids are in the LE phase, while peaks with positions exceeding $0.230 \AA^{-1}$ are found when the lipids are in the LC phase. When the lipids are in the LC phase, the position of the low-angle peak shifts slightly (from 0.23 to $0.26 \AA^{-1}$ ). Its structured rod arises from two scattering centers: one is due to aligned PSS chains and the other to a corrugation of the film/air interface as was shown for PSS with $L_{K}=960$ $\AA^{20,35}$ Here, we find quantitatively the same $Q_{z}$ dependence and conclude that we have a corrugated film/air interface (periodicity $d_{\mathrm{PSS}}$ ), independent of the PSS contour length. The low-angle GID peaks are very similar for all $L_{\mathrm{K}}$ (cf. Figure S6) with one remarkable exception: If $L_{\mathrm{K}}$ is very small $\left(L_{\mathrm{K}}=40,50\right.$, and $80 \AA$ ) and DODA is in the fluid phase, no low-angle peaks are observed.

Wide-Angle Peaks. Besides the low-angle peaks attributed to aligned PSS chains, wide-angle peaks are measured (from 1.3 to $1.45 \AA^{-1}$ ), which are attributed to the ordered alkyl tails of the lipids (cf. Figure 5). They appear at the end of the coexistence region, when the steep increase of the surface pressure starts. Three peaks are observed, one at $Q_{z} \approx 0.1-0.3 \AA^{-1}$ and two overlapping peaks at large $Q_{z}$. On compression, they shift to $Q_{z}$ $\approx 0.73$ and $0.9 \AA^{-1}$, respectively. These features characterize tilted alkyl tails ordered in a oblique lattice, with a decreasing tilt angle $t$ on compression. Therefore, the tilt direction is mainly toward NN (nearest-neighbor) but shifted by the tilt azimuth angle $\psi$ (between $9^{\circ}$ and $12^{\circ}$ ) in the NNN (nextnearest-neighbor) direction. As the distorted shape of the peaks with low $Q_{z}$ already suggests (the $Q_{x y}$ position of maximum intensity decreases on increase of $Q_{z}$ ), the tilt azimuthal angle $\psi$ is broadly distributed, about $15^{\circ}-20^{\circ}$. It does not change on compression. The lattice structure is identical to DODA on clean water or on a PSS solution. ${ }^{20}$

If PSS with different contour lengths is adsorbed, the alkyl tail lattice of the DODA is the same, an oblique lattice with a tilt azimuth distribution (cf. Figure S7). This becomes obvious when the lattice parameters are considered. Figure 6 depicts the tilt angle $t$ with respect to the surface normal and the area of the unit cell, $A_{\text {diffr }}$, calculated from the lattice constants. The lattice parameters are displayed as a function of the DODA molecular area $A$ determined from the isotherm. $A_{\text {diffr }}$ decreases monotonically on monolayer compression. $A_{\text {diffr }}$ is much smaller than $A$ in the LE/LC coexistence region. For very compressed films, $A_{\text {diffr }}$ is only a few percent smaller than $A$. This is expected, since the alkyl lattice contains defects, as 

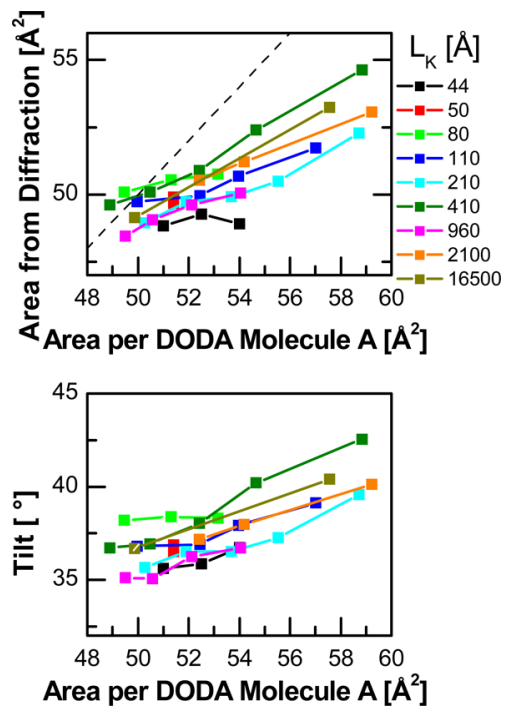

Figure 6. Parameters deduced from the wide-angle GID peaks of the alkyl tails (cf. Figure 5 and Figure S6). Area per DODA molecule $A_{\text {diff }}$ (top) and tilt angle $t$ (bottom) are shown as a function of the molecular area $A$ as determined from the isotherms. The dashed line in the top image is calculated assuming $A=A_{\text {diffr }}$. Solid lines are guides to the eye.

evidenced by the small correlation length (cf Figure 7). ${ }^{1}$ For both $t$ and $A_{\text {diffr' }}$, film to film scatter is observed, but no influence of $L_{\mathrm{K}}$ can be discerned. On monolayer compression, the tilt angle decreases monotonously, from at most $41 \pm 1^{\circ}$ down to $36 \pm 1^{\circ}$. These are large tilt angles, typical for DODA on pure water. ${ }^{20}$

One might argue that large values of $t$ and $A_{\text {diffr }}$ are only observed for contour lengths $L_{\mathrm{K}}$ exceeding $100 \AA$ (cf. Figure 6); when PSS with short contour lengths $\left(L_{\mathrm{K}}=44,50\right.$, and $\left.80 \AA\right)$ adsorbs to DODA, no large values of $t$ and $A_{\text {diffr }}$ are found. This limitation is attributed to the fact that all measurements in Figure 7 are performed at $20{ }^{\circ} \mathrm{C}$. At that temperature, the phase transition pressure $\pi_{\mathrm{c}}$ is high when PSS with low contour lengths $L_{\mathrm{K}}$ is adsorbed (cf. Figures 1 and 3), and the solid phase

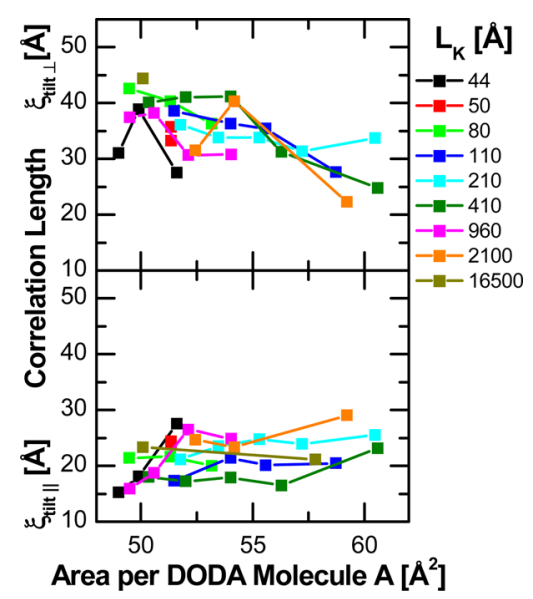

Figure 7. Correlation length $\xi$ derived from peak width of wide-angle diffraction peaks attributed to the alkyl tails of DODA (cf. Figure 5 and Figure S6). At the top is the correlation length roughly perpendicular to the tilt direction (azimuth angle $\Psi \approx 75^{\circ}$ ) depicted and at the bottom roughly parallel to the tilt direction $\left(\Psi \approx 15^{\circ}\right.$ and $\left.\Psi \approx 45^{\circ}\right)$. Lines are guides to the eye. occurs only at low molecular areas $A$. Therefore, $t$ and $A_{\text {diffr }}$ are small, too.

The extension of the positional order within the alkyl chain lattice is measured by the correlation length $\xi_{\text {tilt }}$ (cf. Figure 7). Again, no systematic influence of the PSS contour length can be detected. However, we have to distinguish the difference between the widths of the three large-angle diffraction peaks, indicating a difference in the correlation length (cf. Figure 7), which is related to the tilt azimuth. For all peaks, the peak width and thus the correlation length change a little on monolayer compression. The two overlapping peaks at large $Q_{z}$ (roughly parallel to the tilt direction) start broad; the correlation length $\xi_{\text {tilt,ll }}$ is 5-6 lattice spacings $(20-25 \AA)$ and decreases slightly on monolayer compression to 4 lattice spacings $(18 \AA)$. The correlation length $\xi_{\text {tilt }, \perp}$ roughly normal to the tilt direction is deduced from the single peak at low $Q_{z} \cdot \xi_{\text {tilt, } \perp}$ starts low and increases on compression, from about 5 lattice spacings $(22.5$ $\AA)$ to 9-10 lattice spacings (40-45 $\AA$ ). Always, the correlation length normal to tilt direction is larger. These anisotropic correlation lengths have been observed for many systems. ${ }^{1}$ Theoretical models suggest that the decreased correlation length parallel to the tilt is caused by thermally excited defects, whose nature and concentration depend on the phase of the ordered hydrocarbon chains.

Quantification of PSS Coverage. To quantify the PSS coverage, the chain separation $d_{\mathrm{PSS}}$ is calculated during monolayer compression (cf. Figure 8). $d_{\mathrm{PSS}}$ decreases as a function of DODA molecular area $A$. When the fluid monolayer is compressed, $d_{\mathrm{PSS}}$ falls by almost a factor of 2 , from 50 to 35 $\AA$. When the lipids undergo the phase transition from the fluid to the solid phase, simultaneously a sudden decrease in $d_{\mathrm{PSS}}$ occurs. $d_{\mathrm{PSS}}$ is either larger than $34.8 \AA$ (with fluid lipids) or smaller than $27.2 \AA$ (with solid lipids). When the lipids are in the solid phase, $d_{\mathrm{PSS}}$ decreases slightly (by $15 \%$ ), from 27.2 to 23 Å.

In the next step, the contour length dependence of the lattice parameters is studied. If the lipids are in the LC phase, $d_{\mathrm{PSS}}$ shows no contour length dependence. However, if the lipids are in the fluid phase, a qualitative influence of $L_{\mathrm{K}}$ can be identified: For $L_{\mathrm{K}} \leq 80 \AA$, no two-dimensional lamellar phase exist. For $110 \AA=L_{\mathrm{K}} \leq L_{\mathrm{P}} \approx 210 \AA$, we find that $d_{\mathrm{PSS}}$ decreases on DODA monolayer compression from 45 to $35 \AA$. For $L_{\mathrm{K}}>L_{\mathrm{P}}$ $\approx 210 \AA, d_{\mathrm{PSS}}$ is increased by about $10 \%$.

Finally, we would like to mention that the $d_{\mathrm{PSS}}$ values scatter. The scatter is not due to errors in the LMS fits to the Bragg peaks; in fact, the error bars are smaller than the symbols used in Figure 8. Dominant is the film to film variation, aging $(8 \mathrm{~h}$ equilibration time decreased $\left.d_{\mathrm{PSS}}\right)$ and first or second compression influence the observed peak position. ${ }^{49}$ Please note that each film shows a monotonous decrease of $d_{\mathrm{PSS}}$; however, the starting value and the slope vary slightly.

On monolayer compression, both the lipid molecular area $A$ and $d_{\mathrm{PSS}}$ decrease by a factor of 2 (cf. Figure 8 ). Assuming that all DODA head groups and PSS monomers are charged, the adsorbed PSS in the two-dimensional lamellar phase compensates $80 \%$ of the surface charge of the lipid monolayer. (The area per PSS monomer is calculated according to $A_{\mathrm{PSS} \text {,monomer }}=d_{\mathrm{PSS}} \cdot 2.56 \AA$, with $2.56 \AA$ as the length of a PSS monomer.) Thus, the adsorbed polyelectrolytes in the 2-D lamellar phase almost achieve charge compensation. ${ }^{22}$ The surface charge of the DODA/PSS system is small but still positive. 


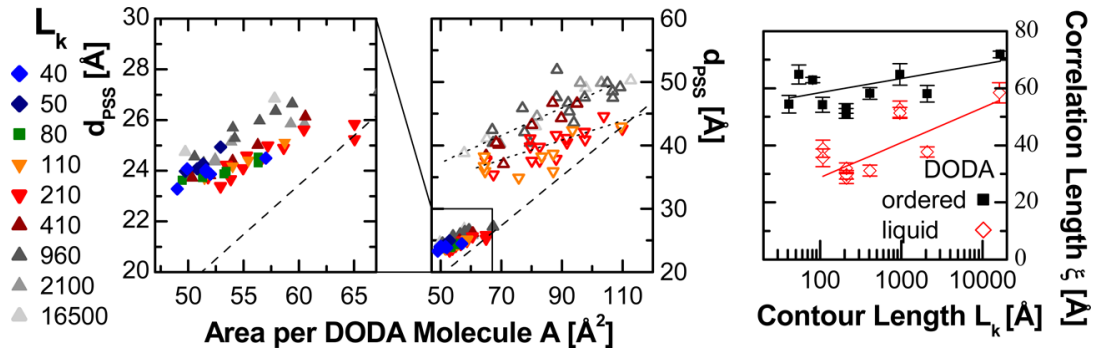

Figure 8. Left and center: the separation between aligned PSS chains $d_{\mathrm{PSS}}$ as a function of the DODA molecular area $A$ for different contour lengths $L_{\mathrm{K}}$ as indicated. The dashed line gives $d_{\mathrm{PSS}}$ assuming charge compensation. Left: $d_{\mathrm{PSS}}$ (full symbols) calculated from the low-angle peaks with the structured rod (cf. Figure 5 and Figure S6); these small values of $d_{\mathrm{PSS}}$ are measured when also high-angle peaks of ordered hydrocarbon tails of the lipids are observed. Centre: $d_{\mathrm{PSS}}$ measured along the isotherm. $d_{\mathrm{PSS}}$ (open symbols) is calculated from the simple low-angle peaks which are observed when high-angle peaks are absent and the lipids are in the fluid phase. The dotted lines are linear fits to the data for $L_{\mathrm{P}} \geq L_{\mathrm{K}}(=110$ and $210 \AA)$ and for $L_{\mathrm{P}}<L_{\mathrm{K}}(=410,960,2100$, and $16500 \AA)$. Right: the correlation length $\xi$ deduced from the width of the low-angle peaks. Each correlation length $\xi$ shown is the average of all measurements taken from the lipid phase indicated. The lines are linear fits. For DODA in the fluid phase, no diffraction peaks are observed for small $L_{\mathrm{K}}(=40,50$, and $80 \AA)$. The PSS concentration is $10^{-5} \mathrm{~mol} / \mathrm{L}$ in monomer units $\left(T=20^{\circ} \mathrm{C}\right)$.
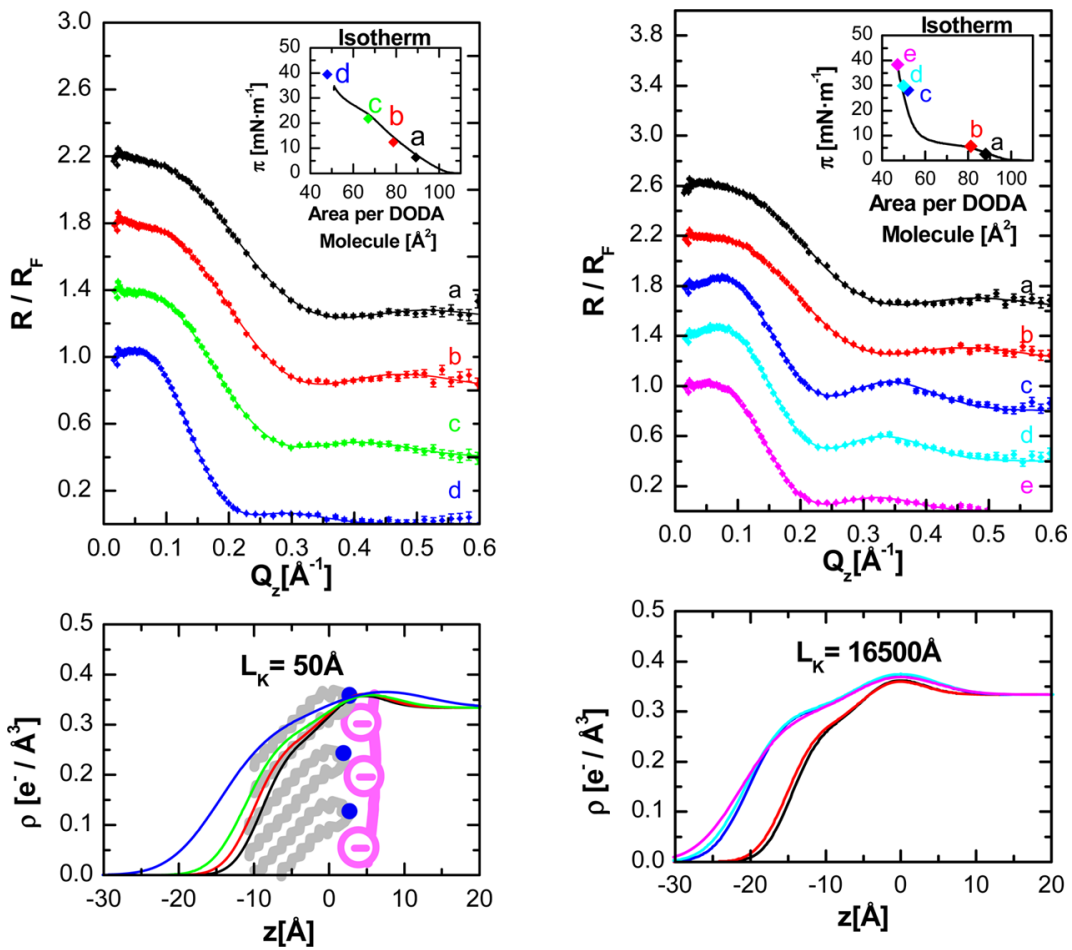

Figure 9. Top: normalized X-ray reflectivity curves convoluted with the instrumental resolution of a DODA monolayer on $0.01 \mathrm{mmol} / \mathrm{L}$ PSS (with respect to the monomer concentration), with the contour length $L_{\mathrm{K}}=50 \AA$ (left) and $16500 \AA$ (right). The molecular areas are indicated in the isotherm shown in the respective insets. For clarity, the X-ray reflectivity curves are displaced vertically. The lines are least-squares fits based on the electron density profiles shown at the bottom. Additionally, the schematic at the bottom left shows the molecular conformation.

Influence of the Lipid Phase on the PSS Phase: PSS Surface Coverage and Correlation Length of the 2-D Lamellar Phase. Already with AFM images, indications of movement of the PSS chains in the two-dimensional lamellar phase adsorbed on a frozen lipid membrane were obtained. ${ }^{19}$ To better describe the 2-D lamellar phase, the positional correlation length $\xi$ is measured. When the lipids are in the fluid phase, the low-angle peaks are very broad in the $Q_{x y}$ direction, suggesting a small correlation length $\xi$ (Figure 5 and Figure S6); when the lipids are in the solid phase, the peaks with the structured rod are narrower in the $Q_{x y}$ direction, indicating an increase of $\xi$. Detailed analysis of the peaks measured along the isotherms shows that for a given lipid phase the correlation length of the low-angle peaks does not change on monolayer compression. Therefore, for each lipid phase and each contour length the correlation length $\xi$ is calculated from the average of all diffraction peaks measured.

When the lipids are in the LC phase, the correlation length $\xi$ of the aligned PSS chains is 60-80 $\AA$. A slight increase of $\xi$ with the contour length is observed. Note that the correlation length $\xi=60-80 \AA$ is larger than the correlation length $\xi_{\text {tilt }}$ of the lipids, both parallel and perpendicular to the tilt direction (which is at most $45 \AA$ ). However, the phase of the lipids influences the correlation length of the PSS chains. When the lipids are in the LE phase, the correlation length $\xi$ is smaller; furthermore, it shrinks on decrease of the contour length $L_{\mathrm{K}}$ drastically, by a factor of 2 from 55 to $30 \AA$.

At first one might say that the lowest correlation length of the PSS chains $\left(\xi=30 \AA\right.$ for $\left.L_{\mathrm{K}}=110 \AA\right)$ is not so small, a similar value was observed for $\xi_{\text {tiltll }}$ (cf. Figure 7 ). However, in 
the 2-D lamellar phase $\xi=30 \AA$ is measured when the chain separation varies between 36 and $44 \AA$. Thus, the correlation length is half the lattice constant; i.e., the lateral order of the two-dimensional lamellar phase is almost lost. For even shorter contour lengths $L_{\mathrm{K}}(\leq 80 \AA)$ no two-dimensional lamellar phase is observed.

If there is a 2-D lamellar order, $\xi$ increases with $L_{\mathrm{K}}$, as expected. ${ }^{50}$ Even though the correlation length $\xi$ of the PSS chains does not change on monolayer compression, the lipid phase does influence $\xi$. Simulations suggest that the coupling between a polymer and a membrane is weaker when the membrane is fluid, due to mobile water molecules and functional groups of the lipid headgroup. ${ }^{51}$ Furthermore, the constant correlation length of the aligned PSS chains during monolayer compression during the LE or LC phase of the lipids suggests that the PSS chains are not subject to steric constraints which would be caused by specific ion bonds.

Out-of-Plane Structure: X-ray Reflectivity. With X-ray reflectivity we obtain the electron density profile perpendicular to the water surface. Therefore, we can determine the surface coverage of PSS when the contour length is so short $\left(L_{\mathrm{K}}=40\right.$, 50 , and $80 \AA$ ) that no diffraction peaks are observed.

The X-ray reflectivity curves of DODA on PSS are shown in Figure 9. Two different contour lengths are used: one very short $\left(L_{\mathrm{K}}=50 \AA\right)$ and one very long $\left(L_{\mathrm{K}}=16500 \AA\right)$. The measurements are taken along the isotherm. At low surface pressures, the reflectivity curve decays slowly until $Q_{z}=0.17$ $\AA^{-1}$, and then it decays faster until $Q_{z}=0.4 \AA^{-1}$; eventually, it is zero. On monolayer compression, zero is reached at lower $Q_{z}$ values, indicating film thickening. When the monolayer is compressed into the solid phase, the flat decay at low $Q_{z}$ changes into a first maximum at $0.075-0.1 \AA^{-1}$; a second maximum can be discerned at high $Q_{z}\left(0.45-0.35 \AA^{-1}\right)$. The minimum shifts to even lower $Q_{z}\left(0.3-0.22 \AA^{-1}\right)$ values, indicating further monolayer thickening. On monolayer compression, the second maximum decreases. This observation is attributed to the increased surface pressure $\pi$, which is equivalent to a decreased surface tension and leads to an increased film/air roughness. (The reflected intensity is proportional to $\exp \left(-\sigma^{2} Q_{z}^{2}\right)$, with $\sigma$ the film/air roughness.)

On increase of the contour length, the reflectivity curves in the LE phase are very similar concerning the minimum and maximum positions, for both lipid phases. The LE/LC phase transition occurs at low $\pi_{\mathrm{c}}$; therefore, more measurements were taken in the LC phase. There are always two maxima. The minimum clearly shifts, suggesting film thickening. This is consistent with the decreasing tilt angle of the alkyl tails, i.e., the increasing thickness of the hydrophobic moiety on film compression. Again, the second maximum decreases on increase of the surface pressure $\pi$.

Reflectivity data were fitted with a simple model consisting of two slabs: one slab for the alkyl chains and one slab for the headgroup together with the adsorbed PSS. The electron density $\rho_{\text {tail }}$ of the tail slab is constrained to give a total number of electrons $E_{\text {tail }}=226 \mathrm{e}^{-}=A \times l_{\text {tail }} \times \rho_{\text {tail }}$, i.e., two alkyl tails with only 14 carbon atoms from the octadecyl chain $\left(l_{\text {tail }}\right.$ denotes the thickness of the alkyl chain slab and $\rho_{\text {tail }}$ denotes its electron density). A common roughness $\sigma$ is used for all three interfaces $\left(\sigma=\sigma_{\text {air-tail }}=\sigma_{\text {tail-head }}=\sigma_{\text {head-subphase }}\right)$. Therefore, all reflectivity curves are fitted with four parameters, namely $l_{\text {tail }}$, $l_{\text {head, }} \rho_{\text {head }}$ and $\sigma$.
The number of PSS monomers $n_{\text {PSS }}$ and water molecules $n_{\mathrm{H}_{2} \mathrm{O}}$ are calculated from the volume $V_{\text {head }}=A \times l_{\text {head }}$ and number of electrons $E_{\text {head }}=A \times l_{\text {head }} \times \rho_{\text {head }}$.

Unknown are the parameters $n_{\mathrm{PSS}}$, the fraction of adsorbed PSS monomers per DODA headgroup, and $n_{\mathrm{H}_{2} \mathrm{O}}$, the number of water molecules in the headgroup. They are calculated with

$$
\begin{aligned}
& V_{\text {head }}=n_{\mathrm{H}_{2} \mathrm{O}} V_{\mathrm{H}_{2} \mathrm{O}}+n_{\mathrm{PSS}} V_{\mathrm{PSS}}+V_{\mathrm{DODA}} \\
& E_{\text {head }}=n_{\mathrm{H}_{2} \mathrm{O}} E_{\mathrm{H}_{2} \mathrm{O}}+n_{\mathrm{PSS}} E_{\mathrm{PSS}}+E_{\mathrm{DODA}}
\end{aligned}
$$

where $V_{\mathrm{H}_{2} \mathrm{O}}=30 \AA^{3}, V_{\mathrm{PSS}}=200 \AA^{3}, V_{\mathrm{DODA}}=290 \AA^{3}$ and $E_{\mathrm{H}_{2} \mathrm{O}}=$ $10 \mathrm{e}^{-}, E_{\mathrm{PSS}}=95 \mathrm{e}^{-}, E_{\mathrm{DODA}}=88 \mathrm{e}^{-}$are the volumes and number of electrons of a water molecule, a PSS monomer, and the DODA headgroup, respectively. A table with the deduced parameters is in the Supporting Information.

Two features are obvious: PSS adsorbs onto DODA in the fluid phase, even if the contour length is short $\left(L_{\mathrm{K}}=50 \AA\right)$. Furthermore, Figure 10 shows that the headgroup contains

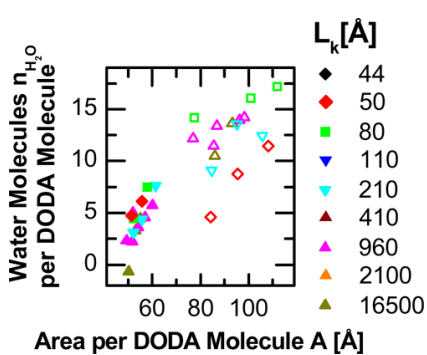

Figure 10. Water molecules per DODA/PSS headgroup measured along the isotherm, as determined from the reflectivity curves shown in Figure 9. The contour length of PSS is varied, as indicated.

more water molecules when the lipids are in the fluid phase. No contour length dependence can be found. This suggests that the adsorbed polyelectrolyte can be described as a cloud of smaller counterions which are connected by the polymer backbone. Apparently, there are few if any specific ion bonds. This observation is consistent with the smaller correlation length $\xi$ of the aligned PSS chains if the lipids are in the LE phase. Therefore, we suggest that the PSS chains adsorb flatly as elastic rods with random distribution (cf. Scheme 1).

Correlation between Structural Data and Thermodynamic Properties. GID data of DODA show always the same LC phase of the lipids: an oblique tilted lattice with an azimuth distribution. It does not change when PSS adsorbs. If the solution contains $0.01 \mathrm{mmol} / \mathrm{L}$ PSS (in monomer units), X-ray reflectivity data demonstrate that always PSS adsorbs, even if no 2-D lamellar phase is observed.

Additionally, GID measurements show that the surface coverage of PSS in the 2-D lamellar phase increases with monolayer compression; both the DODA molecular area $A$ and the separation of the aligned PSS chains $d_{\mathrm{PSS}}$ decrease by a factor of 2. The molecular weight dependence of the surface coverage in the 2-D lamellar phase is beyond resolution (if the lipids are in the LC phase) or weak (if the lipids are in the LE phase). The interchain separation $d_{\mathrm{PSS}}$ is about $10 \%$ larger if the contour length $L_{\mathrm{K}}$ exceeds the persistence length $L_{\mathrm{P}}$ and the surface charge density is low. In other words, long flexible PSS chains show a $10 \%$ smaller surface coverage than short rigid PSS chains, provided the lipids are in the LE phase with its low surface charge density. The correlation length $\xi$ of the aligned PSS chains does depend on (a) the phase of the lipids and (b) 
Scheme 1. Schematic of PSS beneath the Lipid Monolayer, Both for DODA in the Fluid and Solid Phase (Left and Right, Respectively), Side and Top View ${ }^{a}$
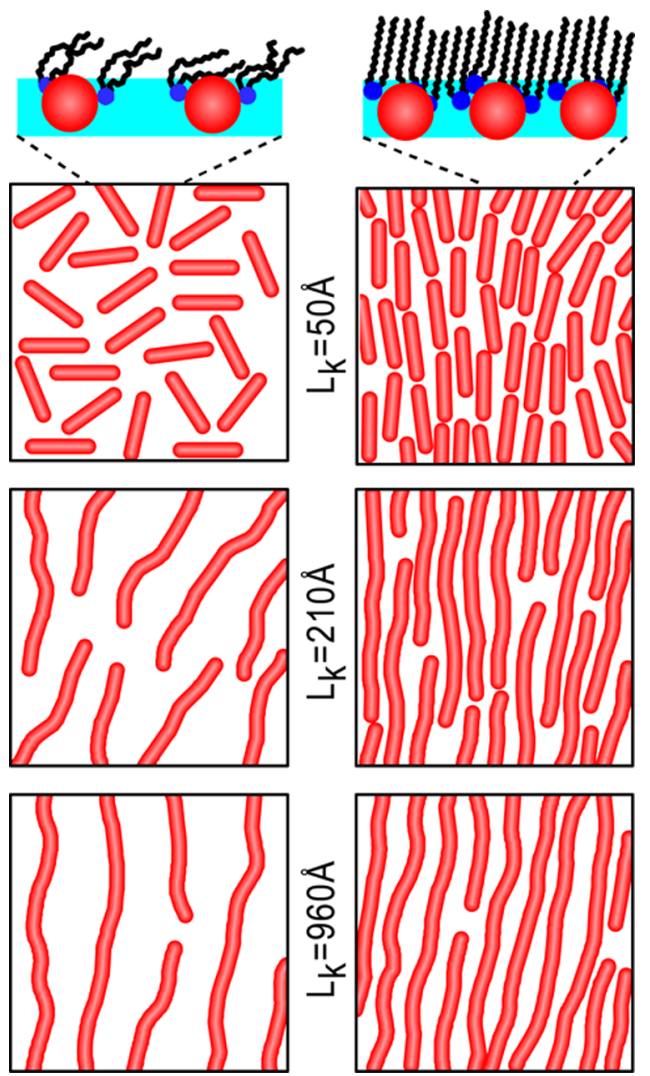

${ }^{a}$ The schematic is drawn to scale. The top panel shows a side view, the three bottom panels top views. Second panel: the length of a stretched PSS chain is $L_{\mathrm{K}}=50 \AA$ corresponding to $N=20$. If the lipids are in the fluid phase, the PSS adsorbs as flat disordered rods. The increased surface charge density of the monolayer in the solid phase causes an increase in PSS surface coverage; now the polyelectrolyte is in the twodimensional lamellar phase. Third and fourth panel: PSS with $L_{\mathrm{K}}=$ 210, $960 \AA(N=80,375)$. Chains align when the contour length is larger than $2 d_{\mathrm{PSS}}$. Therefore, for both lipid phases, PSS is in the twodimensional lamellar phase.

the contour length of PSS. Always, PSS adsorbed to the LE phase shows a shorter correlation length $\xi$ than adsorbed to the LC phase. Furthermore, the correlation length $\xi$ increases with the contour length. The effect is more pronounced when the lipids are in the LE phase. For very small contour lengths $\left(L_{\mathrm{K}}=\right.$ 40,50 , and $80 \AA$ ), PSS adsorbs to lipids in the LE phase in a flatly disordered phase. If the contour length is increased to 110 $\AA$, PSS adsorbs to fluid lipids in the two-dimensional lamellar phase, yet the correlation length is very low $\left(\xi \approx 0.5 d_{\mathrm{PSS}}\right)$.

When the lipids are in the LC phase, their alkyl tails form an oblique lattice and the PSS chains are aligned in the twodimensional lamellar phase. These two ordered phases are independent of each other. There is no relation between the interchain separation and the different lattice spacings of the alkyl tail lattice (cf. Figures 6 and 8). Furthermore, the correlation length of the aligned PSS chains $(\xi=60-80 \AA)$ is larger than the correlation lengths of the alkyl tails in both directions, parallel $\left(\xi_{\text {tilt }, \|}=25-18 \AA\right)$ or perpendicular $\left(\xi_{\text {tilt }, \perp}=\right.$ 22-40 $\AA$ ) to the tilt direction (cf. Figures 7 and 8). When the monolayer in the LC phase is compressed, the correlation length $\xi$ of the PSS chains is constant, while $\xi_{\text {tilt, } \perp}$ increases. These observations suggest that the adsorbed PSS chains can be approximated as a cloud of small counterions which are connected by a polymer backbone. There is no indication of specific binding.

Summarizing the thermodynamic data, the first insight we gain concerns the persistence length $L_{\mathrm{P}}$. The decrease of the transition pressure $\pi_{\mathrm{c}}$ is attributed to the adsorbed polyelectrolytes (cf. Figures 1 and 2). While the contour length is shorter than the persistence length $\left(L_{\mathrm{K}} \leq L_{\mathrm{P}}\right), \pi_{\mathrm{c}}$ decreases with the contour length; however, when the contour length exceeds the persistence length $\left(L_{\mathrm{K}} \geq L_{\mathrm{P}}\right), \pi_{\mathrm{c}}$ is low and constant. In a simplified picture, a stiff chain with $n$ charges binds weakly to $n$ lipids. The PSS chain mediates an attraction between $n$ lipid head groups, and thus decreases the transition pressure $\pi_{\mathrm{c}}$. When the contour length exceeds the persistence length, the PSS chain consists of a collection of hypothetical segments. Each segment mediates an attraction between $n$ lipids. For these large contour lengths $\pi_{c}$ levels off. From the contour length dependence of the transition pressure, we conclude that the persistence length $L_{\mathrm{P}} \approx 210 \AA$.

Changes of the phase transition enthalpy and its temperature dependence are attributed to different interactions of the alkyl tails of the lipid. When long PSS $\left(L_{\mathrm{K}} \geq L_{\mathrm{P}}\right)$ is adsorbed, the values of $\Delta H$ and $\Delta S$ are both weakly temperature dependent and rather small (cf. Figures 3 and 4). This could mean that either the LE phase is more ordered or the LC phase is more disordered. ${ }^{33}$ Considering the identical LC phase observed for all DODA films with adsorbed PSS as well as on pure water, ${ }^{20}$ we conclude that the LC phase is not affected. Therefore, the LE phase is more ordered; presumably the weak electrostatic binding of the lipids to one specific rod (rod length $\leq L_{\mathrm{P}}$ ) reduces the lateral mobility and thus decreases the entropy of lipids in the LE phase. The decreased entropy in the LE phase leads to a reduction of the transition entropy $\Delta S$ and the transition enthalpy $\Delta H$.

For contour lengths much longer than the persistence length $L_{\mathrm{P}}$, PSS acts like a flexible chain, which is confined to the monolayer surface but can be treated with polymer statistics. The decisive variable is the persistence length $L_{\mathrm{P}}$. Theoretically, the in-plane interaction energy (normalized per unit area) is independent of the contour length. ${ }^{28} \mathrm{We}$ observe this effect. When the contour length $L_{\mathrm{K}}$ approaches the persistence length $L_{\mathrm{P}} \approx 210 \AA$, then $\pi_{\mathrm{c}}$ as well as the transition enthalpy $\Delta H$ and the transition entropy $\Delta S$ level off.

Additionally, the contour length dependence of $\Delta H$ shows one additional feature: a maximum at $L_{\mathrm{K}}=80 \AA$ (cf. Figure 4). This maximum of $\Delta H$ correlates with the phases of the flatly adsorbed PSS. When $L_{\mathrm{K}}$ is very small $\left(L_{\mathrm{K}}=40,50\right.$, and $\left.80 \AA\right)$, PSS adsorbs in an isotropic phase, as disordered rods (cf. Scheme 1). When this monolayer is compressed, two phase transitions occur simultaneously: the LE/LC transition of the lipids and the isotropic/2-D lamellar phase transition of adsorbed PSS. The transition enthalpy $\Delta H$ increases with rod length. The longest PSS chains, with which these two simultaneous phase transitions occur, have a contour length of $80 \AA$. On further increase of $L_{\mathrm{K}}$, PSS adsorbs already to fluid lipids in the 2-D lamellar phase. Then, the LE/LC phase transition is accompanied by a sudden decrease of the interchain separation $d_{\mathrm{PSS}}$ (cf. Figure 8), and the transition enthalpy decreases with the contour length $L_{\mathrm{K}}$, until the contour length exceeds the persistence length $L_{\mathrm{P}}$. 


\section{CONCLUSION}

A combination of thermodynamic and X-ray data is used to investigate the adsorption of the strong anionic polyelectrolyte PSS (0.01 $\mathrm{mmol} / \mathrm{L}$ in monomer units) to the oppositely charged monolayer DODA at the air/water interface. The aim of this study is to shed some light on the polyelectrolytemonolayer interaction.

We distinguish (1) the effect of the monolayer on the conformation and phase of the adsorbed polyelectrolyte (PSS) and (2) the effect of the adsorbed PSS on the phases and phase transitions of the lipid monolayer. The effect of the monolayer on PSS is straightforward: PSS adsorbs in the two-dimensional lamellar phase, and the interchain separation $d_{\mathrm{PSS}}$ is independent of the contour length (LC phase of the lipids) or depends slightly on the contour length (LE phase of the lipids). Therefore, we conclude that the surface charge density of the monolayer determines the interchain separation $d_{\mathrm{PSS}}$. An exception is very low molecular weight PSS (contour length 80 $\AA$ or less) and very low surface charge density, i.e., LE phase of the lipids. Then, no two-dimensional lamellar phase is found, but a flat isotropic phase; the flatly adsorbed rigid chains are disordered (cf. Scheme 1).

The effect of the adsorbed PSS on the monolayer is more involved: PSS adsorption has a condensing effect on the monolayer. While the PSS chain is short and can be described as an elastic rod, the transition pressure $\pi_{\mathrm{c}}$ decreases with increasing rod length. When the contour length $L_{\mathrm{K}}$ of the adsorbing PSS exceeds the persistence length $L_{\mathrm{P}}$, then the transition pressure $\pi_{\mathrm{c}}$ is low and constant.

Additionally, PSS adsorption affects the in-plane interaction of the lipids: In a simplified picture, a PSS molecule with $n$ charges neutralizes $n$ lipid molecules. With increasing $n$, we find a decreased entropy change $\Delta S$ at the LE/LC phase transition, which may be explained by the partial immobilization of $n$ fluid lipids attracted electrostatically to a PSS molecule with $n$ charged monomers. We suggest that the number of partially immobilized lipids is constant when the contour length exceeds the persistence length. Thus, the contour length dependence of the transition entropy $\Delta S$ and the transition enthalpy $\Delta H$ can be explained, provided PSS is in the two-dimensional lamellar phase for all lipid phases.

However, for contour lengths equal to or below $80 \AA$, two simultaneous disordered/ordered phase transitions occur on monolayer compression: the LE/LC transition of the lipids and the isotropic/2-dimensional lamellar transition of the rigid PSS chains. In this case, an increase of the contour length induces an increase in the transition enthalpy.

To summarize, the contour length dependence of the transition enthalpy is complex, but it can be understood: (a) For very short contour lengths ( $80 \AA$ or smaller) the PSS isotropic/2-dimensional lamellar and the lipid LE/LC phase transitions occur simultaneously, the transition enthalpy increases with the contour length of PSS. (b) Longer PSS chains still in the rod-like regime (with a contour length larger than $80 \AA$ ) align when the lipids are in the LE phase. The transition enthalpy decreases until the contour length reaches the persistence length $(210 \AA)$. (c) When the contour length of the adsorbed PSS exceeds its persistence length, then the transition entropy is low and constant.

Finally, we would like to emphasize that the combination of structural and thermodynamic data is a very powerful approach. Grazing incidence diffraction allows to determine the surface coverage and especially the phase of the adsorbed polyelectrolyte. This knowledge makes it possible to gain new insight into the polyelectrolyte-monolayer interaction.

\section{ASSOCIATED CONTENT}

\section{Supporting Information}

A table which lists the molecular weight, contour length, and degree of polymerization of all PSS used; additional isotherms at different temperatures, the pressure dependence of thermodynamic parameters, all GID diffraction peaks, and the parameter tables derived from the X-ray reflectivity measurements. This material is available free of charge via the Internet at http://pubs.acs.org.

\section{AUTHOR INFORMATION}

\section{Corresponding Author}

*E-mail helm@uni-greifswald.de (C.A.H.).

\section{Notes}

The authors declare no competing financial interest.

\section{ACKNOWLEDGMENTS}

We thank HASYLAB at DESY, Hamburg, Germany, for beam time and for providing all necessary facilities. Timely discussions with Burkhard Dünweg were illuminating; the comments of Stephan Block were helpful. Financial support of the DFG (He 1616/14-1) is appreciated.

\section{REFERENCES}

(1) Kaganer, V. M.; Möhwald, H.; Dutta, P. Structure and phase transitions in Langmuir monolayers. Rev. Mod. Phys. 1999, 71, 779819

(2) Möhwald, H. Phospholipid and phospholipid protein monolayers at the air/water interface. Annu. Rev. Phys. Chem. 1990, 41, 441-476.

(3) Knobler, C. M.; Desai, R. C. Phase transitions in monolayers. Annu. Rev. Phys. Chem. 1992, 43, 207-236.

(4) McConnell, H. M. Structures and transitions in lipid monolayers at the air-water-interface. Annu. Rev. Phys. Chem. 1991, 42, 171-195.

(5) Majewski, J.; Kuhl, T. L.; Kjaer, K.; Smith, G. S. Packing of ganglioside-phospholipid monolayers: An X-ray diffraction and reflectivity study. Biophys. J. 2001, 81, 2707-2715.

(6) Brezesinski, G.; Dietrich, A.; Struth, B.; Böhm, C.; Bouwman, W. G.; Kjaer, K.; Mohwald, H. Influence of ether linkages on the structure of double-chain phospholipid monolayers. Chem. Phys. Lipids 1995, 76, $145-157$.

(7) Dahmen-Levison, U.; Brezesinski, G.; Möhwald, H. Specific adsorption of PLA 2 at monolayers. Thin Solid Films 1998, 327, 616620.

(8) Brockman, H. Lipid monolayers: why use half a membrane to characterize protein-membrane interactions? Curr. Opin. Struct. Biol. 1999, 9, 438-443.

(9) Brezesinski, G.; Möhwald, H. Langmuir monolayers to study interactions at model membrane surfaces. Adv. Colloid Interface Sci. 2003, 100, 563-584.

(10) Holten-Andersen, N.; Henderson, J. M.; Walther, F. J.; Waring, A. J.; Ruchala, P.; Notter, R. H.; Lee, K. Y. C. KL4 peptide induces reversible collapse structures on multiple length scales in model lung surfactant. Biophys. J. 2011, 101, 2957-2965.

(11) Paiva, D.; Brezesinski, G.; Pereira, M. D.; Rocha, S. Langmuir monolayers of monocationic lipid mixed with cholesterol or fluorocholesterol: DNA adsorption studies. Langmuir 2013, 29, 1920-1925.

(12) Meijere, K. d.; Brezesinski, G.; Kjaer, K.; Möhwald, H. Structure studies in coupled lipid-polyelelctrolyte monolayers with diluted charge densities. Langmuir 1998, 14, 4204-4209. 
(13) Szilagyi, I.; Trefalt, G.; Tiraferri, A.; Maroni, P.; Borkovec, M. Polyelectrolyte adsorption, interparticle forces, and colloidal aggregation. Soft Matter 2014, 10, 2479-502.

(14) Berndt, P.; Kurihara, K.; Kunitake, T. Adsorption of poly(styrenesulfonate) onto an ammonium monolayer on mica: A surface forces study. Langmuir 1992, 8, 2486-2490.

(15) Lowack, K.; Helm, C. A. Molecular mechanisms controlling the self-assembly process of polyelectrolyte multilayers. Macromolecules 1998, 31, 823-833.

(16) Decher, G. Fuzzy nanoassemblies: Toward layered polymeric multicomposites. Science 1997, 277, 1232-1237.

(17) Schönhoff, M. Self-assembled polyelectrolyte multilayers. Curr. Opin. Colloid Interface Sci. 2003, 8, 86-95.

(18) Hammond, P. T. Form and function in multilayer assembly: New applications at the nanoscale. Adv. Mater. 2004, 16, 1271-1293.

(19) Gromer, A.; Rawiso, M.; Maaloum, M. Visualization of hydrophobic polyelectrolytes using atomic force microscopy in solution. Langmuir 2008, 24, 8950-8953.

(20) Günther, J. U.; Ahrens, H.; Helm, C. A. Two-dimensional lamellar phase of poly(styrene sulfonate) adsorbed onto an oppositely charged lipid monolayer. Langmuir 2009, 25, 1500-1508.

(21) Netz, R. R.; Andelman, D. Neutral and charged polymers at interfaces. Phys. Rep. 2003, 380, 1-95.

(22) Netz, R. R.; Joanny, J.-F. Adsorption of semiflexible polyelectrolytes on charged planar surfaces: Charge compensation, charge reversal, and multilayer formation. Macromolecules 1999, 32, 9012-9025.

(23) van de Steeg, H. G. M.; Stuart, M. A. C.; Keizer, A. d.; Bijsterbosch, B. H. Polyelectrolyte adsorption: A subtle balance of forces. Langmuir 1992, 8, 2538-2546.

(24) Stuart, M. A. C. Polyelectrolytes on Solid Surfaces. In Short and Long Chains at Interfaces, Proceeding of the XXXth Rencontres de Moriond; Daillant, J., Guenoun, P., Marques, C., Muller, P., Vân, J. T. T., Eds.; Editions Frontières: Gif-sur-Yvette, France, 1996; pp 1-12.

(25) Messina, R. Electrostatics in soft matter. J. Phys.: Condens. Matter 2009, 21, 18.

(26) Ivanov, V. A.; Rodionova, A. S.; Martemyanova, J. A.; Stukan, M. R.; Muller, M.; Paul, W.; Binder, K. Conformational properties of semiflexible chains at nematic ordering transitions in thin films: A Monte Carlo simulation. Macromolecules 2014, 47, 1206-1220.

(27) Ni, S. L.; Lee, W.; Li, B. B.; Esker, A. R. Thermodynamics of the liquid expanded to condensed phase transition of poly(L-lactic acid) in Langmuir monolayers. Langmuir 2006, 22, 3672-3677.

(28) Odijk, T. On the statistics and dynamics of confined or entangled polymers. Macromolecules 1983, 16, 1340-1344.

(29) Vink, R. L. C. The isotropic-to-nematic transition in a twodimensional fluid of hard needles: a finite-size scaling study. Eur. Phys. J. B 2009, 72, 225-231.

(30) Kahlitz, P.; Stark, H. Phase ordering of hard needles on a quasicrystalline substrate. J. Chem. Phys. 2012, 136, 10.

(31) Albrecht, O.; Gruler, H.; Sackmann, E. Polymorphism of phospholipid monolayers. J. Phys. (Paris) 1978, 39, 301-313.

(32) Helm, C. A.; Laxhuber, L.; Lösche, M.; Möhwald, H. Electrostatic interactions in phospholipid membranes: Influence of monovalent ions. Colloid Polym. Sci. 1986, 264, 46-55.

(33) Yue, X.; Steffen, P.; Dobner, B.; Brezesinski, G.; Möhwald, H. Monolayers of mono-and bipolar palmitic acid derivatives. Colloids Surf., A 2004, 250, 57-65.

(34) Kjaer, K.; Als-Nielsen, J.; Helm, C. A.; Laxhuber, L. A.; Möhwald, H. Ordering in lipid monolayers studied by synchrotron Xray diffraction and fluorescence microscopy. Phys. Rev. Lett. 1987, 58, 2224-2227.

(35) Ahrens, H.; Papastavrou, G.; Schmidt, M.; Helm, C. A. Synchrotron X-ray diffraction and reflection studies of a polymacromonomer monolayer at the air-water interface: Transition from straight aligned molecules to homogeneous layer. J. Phys. Chem. B 2004, 108, 16870-16876.
(36) Baltes, H.; Schwendler, M.; Helm, C. A.; Möhwald, H. Tail and head group interactions in phospholipid monolayers. J. Colloid Interface Sci. 1996, 178, 135-143.

(37) Braslau, A.; Deutsch, M.; Pershan, P. S.; Weiss, A. H.; AlsNielsen, J.; Boch, J. Surface roughness of water measured by X-ray reflectivity. Phys. Rev. Lett. 1985, 58, 2457-2470.

(38) Helm, C. A.; Möhwald, H.; Kjaer, K.; Als-Nielsen, J. Phospholipid monolayer density distribution perpendicular to the water surface. A synchrotron X-ray reflection study. Europhys. Lett. 1987, 4, 697-703.

(39) Pedersen, J. S. Model-independent determination of surface scattering-length-density profile form specular reflectivity data. J. Appl. Crystallogr. 1992, 25, 129-145.

(40) Pedersen, J. S.; Hamley, I. W. Analysis of neutron and X-ray reflectivity data. II. Constrained least-square methods. J. Appl. Crystallogr. 1994, 27, 36-49.

(41) Maltseva, E.; Shapovalov, V. L.; Möhwald, H.; Brezesinski, G. Ionization state and structure of L-1,2-dipalmitoylphosphatidylglycerol monolayers at the liquid/air interface. J. Phys. Chem. B 2006, 110, 919-926.

(42) Levental, I.; Janmey, P. A.; Cebers, A. Electrostatic contribution to the surface pressure of charged monolayers containing polyphosphoinositides. Biophys. J. 2008, 95, 1199-1205.

(43) Wesemann, A.; Steitz, R.; Ahrens, H.; Förster, S.; Helm, C. A. Internal interface of a compressed PEE-PEO diblock copolymer monolayer. Langmuir 2003, 19, 709-716.

(44) Ahrens, H.; Baekmark, T. R.; Merkel, R.; Schmitt, J.; Graf, K.; Raiteri, R.; Helm, C. A. Hydrophilic/hydrophobic nanostripes in lipopolymer monolayers. ChemPhysChem 2000, 1, 101-106.

(45) Bernardini, C.; Stoyanov, S. D.; Stuart, M. A. C.; Arnaudov, L. N.; Leermakers, F. A. M. Polymers at the water/air interface, surface pressure isotherms, and molecularly detailed modeling. Langmuir 2010, 26, 11850-11861.

(46) Berg, F.; Block, S.; Drache, S.; Hippler, R.; Helm, C. A. Effects of reactive oxygen species on single polycation layers. J. Phys. Chem. B 2013, 117, 8475-8483.

(47) Cahill, B. P.; Papastavrou, G.; Koper, G. J. M.; Borkovec, M. Adsorption of poly(amido amine) (PAMAM) dendrimers on silica: Importance of electrostatic three-body attraction. Langmuir 2008, 24 $465-473$.

(48) Adamczyk, Z.; Warszynski, P. Role of electrostatic interactions in particle adsorption. Adv. Colloid Interface Sci. 1996, 63, 41-149.

(49) Helm, C. A.; Möhwald, H.; Kjaer, K.; Als-Nielsen, J. Phospholipid monolayers between fluid and solid states. Biophys. J. 1987, 52, 381-391.

(50) Rubinstein, M.; Colby, R. H. Polymer Physics; Oxford University Press: New York, 2003.

(51) Hirose, Y.; Komura, S.; Andelman, D. Coupled modulated bilayers: A phenomenological model. ChemPhysChem 2009, 10, 28392846. 